A fuzzy convolution model for radiobiologically optimized radiotherapy margins

This article has been downloaded from IOPscience. Please scroll down to see the full text article.

2010 Phys. Med. Biol. 553219

(http://iopscience.iop.org/0031-9155/55/11/015)

View the table of contents for this issue, or go to the journal homepage for more

Download details:

IP Address: 194.176.105.35

The article was downloaded on 18/05/2010 at 09:05

Please note that terms and conditions apply. 


\title{
A fuzzy convolution model for radiobiologically optimized radiotherapy margins
}

\author{
Bongile Mzenda $^{1}$, Mir Hosseini-Ashrafi $^{1}$, Alex Gegov $^{2}$ and \\ David J Brown ${ }^{3}$ \\ ${ }^{1}$ Radiotherapy Physics Department, Queen Alexandra Hospital, Portsmouth Hospitals NHS \\ Trust, Portsmouth, UK \\ ${ }^{2}$ School of Computing, University of Portsmouth, Buckingham Building, Lion Terrace, \\ Portsmouth, PO1 3HE, UK \\ ${ }^{3}$ Institute of Industrial Research, University of Portsmouth, Mercantile House, Portsmouth, \\ PO1 2EG, UK \\ E-mail: bongile.mzenda@porthosp.nhs.uk
}

Received 16 January 2010, in final form 15 April 2010

Published 17 May 2010

Online at stacks.iop.org/PMB/55/3219

\begin{abstract}
In this study we investigate the use of a new knowledge-based fuzzy logic technique to derive radiotherapy margins based on radiotherapy uncertainties and their radiobiological effects. The main radiotherapy uncertainties considered and used to build the model were delineation, set-up and organ motion-induced errors. The radiobiological effects of these combined errors, in terms of prostate tumour control probability and rectal normal tissue complication probability, were used to formulate the rule base and membership functions for a Sugeno type fuzzy system linking the error effect to the treatment margin. The defuzzified output was optimized by convolving it with a Gaussian convolution kernel to give a uniformly varying transfer function which was used to calculate the required treatment margins. The margin derived using the fuzzy technique showed good agreement compared to current prostate margins based on the commonly used margin formulation proposed by van Herk et al (2000 Int. J. Radiat. Oncol. Biol. Phys. 47 1121-35), and has nonlinear variation above combined errors of $5 \mathrm{~mm}$ standard deviation. The derived margin is on average $0.5 \mathrm{~mm}$ bigger than currently used margins in the region of small treatment uncertainties where margin reduction would be applicable. The new margin was applied in an intensity modulated radiotherapy prostate treatment planning example where margin reduction and a dose escalation regime were implemented, and by inducing equivalent treatment uncertainties, the resulting target and organs at risk doses were found to compare well to results obtained using currently recommended margins.
\end{abstract}

(Some figures in this article are in colour only in the electronic version) 


\section{Introduction}

The treatment of cancer using external beam radiotherapy involves a delicate balance between irradiating the entire tumour to kill all the cancerous cells and sparing the surrounding healthy tissues and organs through which the incident radiation beam traverses. This is further complicated by the presence of organ motion of the tumour volume, in particular during treatments, which can be due to the effects of breathing, bowel filling, swallowing or heart beat induced, as well as daily set-up variations in the radiotherapy process which all lead to the variability in the actual dose received compared to the planned dose. Also the extent of microscopic tumour growth is undetectable using current imaging techniques, leading to the uncertainty in treatment volume outlining. The use of treatment margins provides a viable solution to this predicament. A number of recommendations are available for the derivation of margins for use in radiotherapy treatment planning, including the International Commission on Radiation Units and Measurements (ICRU) reports (ICRU 50 1993, ICRU 62 1999) and other formulations (Stroom et al 1999, van Herk et al 2000). New techniques in radiotherapy such as image guided radiotherapy (IGRT) allow for the reduction of the organ motion and set-up errors encountered in the radiotherapy process; however, some residual errors still remain, making the continued use of margins necessary.

The fundamental concepts of tumour control probability (TCP) and normal tissue complication probability (NTCP) describe the probabilities of killing all tumour cells in a volume, and the damage to normal tissues and critical organs, respectively. The therapeutic gain, or probability of cure without complications, is obtained by calculating the uncomplicated tumour control probability (UTCP) (Wolbarst et al 1980), which in the simplified form is approximated by

$$
\mathrm{UTCP}=\mathrm{TCP}(1-\mathrm{NTCP}) .
$$

The relationship between the radiobiological parameters and radiotherapy margins is not well established and difficult to quantify mathematically. In this study we propose the use of a fuzzy logic-based technique to link these variables. A fuzzy technique was first used by Waschek et al (1997) for the derivation of radiotherapy margins. Their technique relied on expert knowledge to derive the clinical target volume (CTV) margin. Their study however did not consider the effects of organ motion and set-up errors leading to the derivation of margins between the CTV and the planning target volume (PTV), i.e. CTV-PTV margins, which is addressed in our study. Also, due to the subjective nature of expert knowledge, particularly for radiotherapy margins where individual delineation errors are considered, the results from such a system are strongly dependent on the input, and will vary significantly between different observers. In our study, the input rules are based on a statistical simulation technique, thus removing the subjective nature of inter-observer variations.

Treatment margins have previously been derived based on radiobiological considerations by a number of investigators. Lind et al (1993) derived an analytical formula dependent on the fractionation, dose homogeneity and the vicinity of the dose limiting structures to the target volume in determining the margin sizes. A linear quadratic model for cell survival was also incorporated (Löf et al 1995) in developing an algorithm to obtain field sizes leading to adequate tumour dose coverage. On the other hand Stavrev et al (1996) used a one-dimensional model and TCP distributions for target volume selection; however, they did not consider the effects of normal tissue complications in their study.

The commonly used ICRU formulations (ICRU 50 1993, ICRU 62 1999) and the margin recipe proposed by van Herk et al (2000) do not explicitly consider the effects of the surrounding organs at risk when deriving CTV-PTV margins for use in treatment planning. 
These margins are derived based on geometrical considerations only and do not consider the radiobiological effects of the tumour and surrounding critical organs. The aim of this study is to consider both geometrical and radiobiological factors in determining the PTV margins for use in prostate radiotherapy planning. The implementation of a novel application of the fuzzy technique specifically to derive CTV-PTV margins in external beam radiotherapy is presented in this work. Delineation errors tend to be underestimated and sometimes omitted in some radiotherapy margin formulations but are included in this study. The published margin formulations assume a linear relationship between the PTV margin and radiotherapy errors. This study also tests the validity of this linear relation assumption. The proximity and influence of surrounding critical structures is taken into account in the CTV-PTV margin derivation by including NTCP effects.

Fuzzy logic was chosen for use in this study because the parameters for tumour control (TCP), damage to healthy tissues (NTCP), delineation errors, organ motion, set-up errors and the required margin cannot be combined easily using mathematical formulations. This may be one of the reasons why current margin formulations use only geometrical considerations for margin formulations as these can be combined using statistical techniques. Fuzzy logic's linguistic rules and membership functions offer a modelling methodology to link the above geometrical and radiobiological parameters. For a nonlinear system as described in this study the Sugeno type fuzzy system is considered more suitable than, for example, a Mamdani-type fuzzy system for modelling purposes (Kim et al 1998, Ying 1998). Fuzzy logic also has the potential to be combined with existing algorithms in radiotherapy planning, leading to intelligent solutions to the complexities encountered in current and emerging radiotherapy treatment techniques.

New IMRT delivery technologies are becoming increasingly available for radiotherapy use, including volumetric dynamic arc delivery systems and radiosurgery systems. These techniques allow for precise dose conformity and delivery, allowing for dose escalation. This results in areas of high dose gradients usually lying close to critical organs. Treatment margins used in such applications need careful optimization to achieve the required tumour cure and sparing of critical organs. The application of the derived fuzzy margin to such a clinical situation is assessed in this study.

Organ deformation, including the effects of tumour shrinkage during radiotherapy treatment, has not been covered in the scope of this work; however, using fuzzy logic it is possible to include such effects by using deformable boundary models (Kobashi et al 2009) which can be incorporated into our model. Also for some treatment sites, such as head \& neck, the uncertainty due to delineation errors can be larger than the required CTV-PTV margin; hence, our study was confined to the prostate site where this variability is generally small. It should also be noted that the present study does not consider the effects of different fractionation regimes. A single fixed fractionation scheme is assumed throughout.

\section{Methods and materials}

\subsection{Overview of the modelling technique}

A number of iterative treatment planning, statistical, simulation and computational steps were performed to develop and validate the margin model used in this study. Figure 1 illustrates the steps taken to derive CTV-PTV margins based on a Sugeno-type knowledge-based fuzzy logic technique.

The above steps are described in more detail in the proceeding sections. 
Produce treatment plans for prostate cancer for typical small, medium and large target volumes using varying CTV-PTV margins (0-18 mm). Calculate baseline TCP and NTCP values.

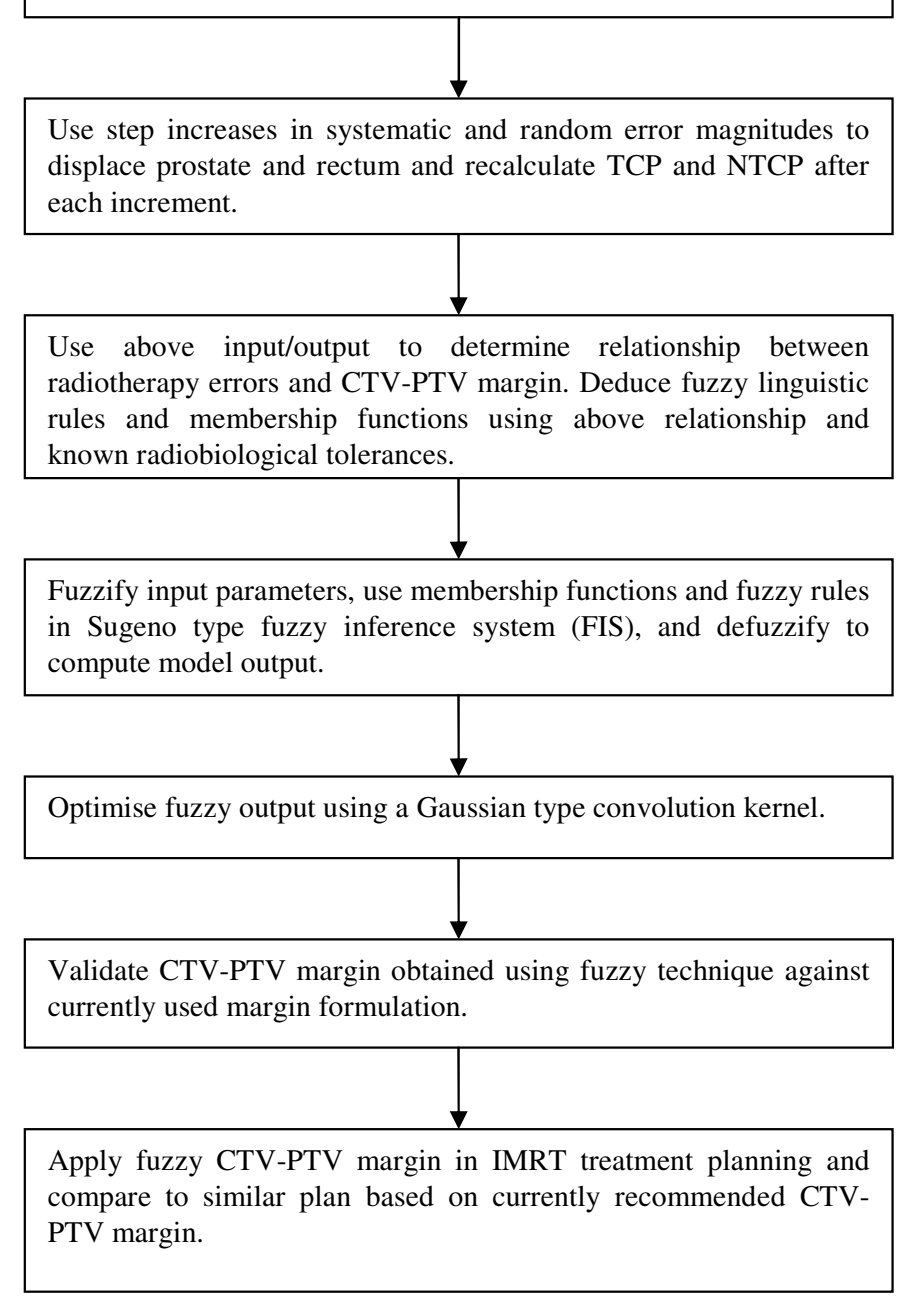

Figure 1. Illustration of the steps taken to model and validate a fuzzy logic-based technique for the derivation and validation of CTV-PTV margins for the prostate.

\subsection{Treatment planning and computation of radiobiological parameters}

Five conformal treatment plans for prostate gross tumour volumes (GTVs) ranging from 33 to $72 \mathrm{~cm}^{3}$ were generated on the CMS $(\mathrm{XiO})$ treatment planning system using the superposition algorithm. A 2 Gy per fraction prescription using $6 \mathrm{MV}$ photons was used to give a total dose of $70 \mathrm{~Gy}$ in 35 fractions. The baseline plans were produced with a CTV only margin, i.e. no CTV-PTV margin $(M)$ grown. For each of these plans uniform CTV-PTV margins $M$ of 0,3 , $5,8,10,12,15$ and $18 \mathrm{~mm}$ were outlined. Treatment plans were then produced for each of these margins with the $95 \%$ isodose level covering the PTV. This range of margins was chosen 
Table 1. Parameters used for prostate TCP and for rectum NTCP modelling for a 2 Gy per fraction dose prescription.

\begin{tabular}{lclll}
\hline Structure & $a$ & $\mathrm{D}_{50} / \mathrm{TD}_{50}(\mathrm{~Gy})$ & $\gamma 50$ & $m$ \\
\hline Prostate & -12 & 46.3 & 0.95 & - \\
Rectum & 8.33 & 80 & - & 0.15 \\
Bladder & 2 & 80 & - & 0.11 \\
\hline
\end{tabular}

to permit the fuzzy model to establish the clinically usable range of margins and to determine an upper margin limit based on the tolerance of the critical organs. All the plans used the same CTV margin size which for the case of the prostate is the same as the GTV.

The TCP values for the prostate CTV were calculated based on the equivalent uniform dose (EUD) radiobiological modelling following the recommendations of Gay and Niermierko (2007). The EUD is defined as the uniform dose distribution giving an equivalent survival fraction to that of a heterogeneous dose distribution (Wu et al 2002). The EUD-based TCP is described by the relationship

$$
\mathrm{TCP}=\frac{1}{1+\left(\frac{D_{50}}{\mathrm{EUD}}\right)^{4 \gamma_{50}}},
$$

where $D_{50}$ is the dose to control $50 \%$ of the tumour following homogenous irradiation, $\gamma_{50}$ describes the slope of the dose-response curve, and the EUD is given by

$$
\mathrm{EUD}=\left(\sum_{i=1}\left(v_{i} D_{i}^{a}\right)\right)^{\frac{1}{a}},
$$

where $a$ is a parameter specific to the normal structure or tumour and $v_{i}$ represents the partial volume receiving the dose $D_{i}$. The generalized mean dose is used in this case as a descriptor of the EUD.

The parameters shown in table 1, based on the recommendations of various prostate cancer studies (Burman et al 1991, Emami et al 1991, Ragazzi et al 1997) were used in the TCP calculation.

Similarly the NTCP values for the rectum were calculated using the parameters in table 1 according to the Lyman-Kutcher-Burman (LKB) model (Burman et al 1991), using the equation

$$
\mathrm{NTCP}=\frac{1}{\sqrt{2 \pi}} \int_{0}^{x} \mathrm{e}^{(-x 2 / 2)} \mathrm{d} x
$$

where

$$
x=\frac{\mathrm{EUD}-T D_{50}}{m \cdot T D_{50}}
$$

and $T D_{50}$ is the tolerance dose for a $50 \%$ complication rate for a specific time period whilst $m$ is a unitless parameter accounting for the volume effect; this determines the steepness of the model curve.

Using equations (2)-(5) the baseline tumour control probability $\left(\mathrm{TCP}_{o}\right)$ and baseline normal tissue complication probability $\left(\mathrm{NTCP}_{o}\right)$ values were calculated for all the plans and margin sizes $M$ used in the study. 


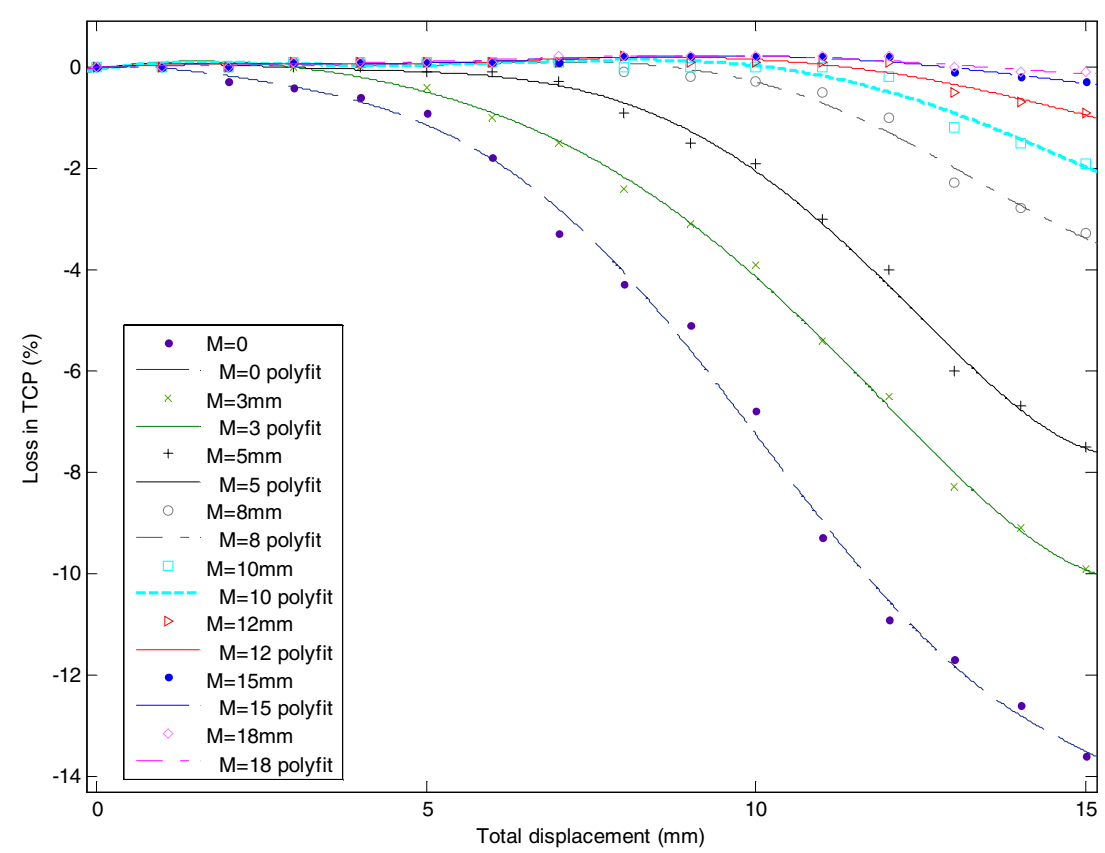

Figure 2. Effect of combined delineation, organ motion and set-up errors on prostate $\Delta \mathrm{TCP}$, based on a 2 Gy per fraction dose prescription, for one patient's treatment plans using incremental margins $M$.

\subsection{Effects of organ motion and set-up errors on TCP and NTCP}

The magnitude of prostate tumour displacement has been found to be up to $15 \mathrm{~mm}$ in various studies using real patients (Miralbell et al 1998, Sur et al 1993). The uncertainty in prostate CTV delineation evaluated locally was found to have a mean difference of the order of $2.5 \mathrm{~mm}$ standard deviation (s.d.); this is of the same order of magnitude as reported in other studies (Seddon et al 2000, Jones et al 1995). Combined organ motion and set-up errors in $0.5 \mathrm{~mm}$ stepsizes up to a magnitude of $15 \mathrm{~mm}$, as well as delineation errors in $0.1 \mathrm{~mm}$ steps were used in our study to calculate the change in the radiobiological measures of response TCP and NTCP. To do this the plans were imported into a Matlab-based tool where incremental translations based on the combined error around the centre of mass of the prostate CTV and bladder were performed. In this manner the organ was displaced with respect to the dose distribution. The resulting total organ dose was the summation of the recalculated dose to each voxel following the shift. The corresponding new radiobiological measures of response $\mathrm{TCP}_{i}$ and $\mathrm{NTCP}_{i}$ were calculated after each step and this was used to deduce the loss of prostate tumour control $\triangle \mathrm{TCP}$ (calculated as the percentage difference between $\mathrm{TCP}_{i}$ and $\mathrm{TCP}_{o}$ ) and the increase in rectal complications $\triangle \mathrm{NTCP}$ (calculated as the percentage difference between $\mathrm{NTCP}_{i}$ and $\mathrm{NTCP}_{o}$ ). This was performed for all $M$ margins used in the treatment plans.

Figure 2 shows that, as expected, the increase in treatment margin $M$ resulted in the gain in TCP. Increasing the errors resulted in the increased loss of TCP. For combined errors with magnitude of up to $15 \mathrm{~mm}$ used in our study the TCP was found to decrease by up to $14 \%$ when no CTV-PTV margin was used; up to $7.5 \%$ for the $5 \mathrm{~mm}$ margin; up to $2.0 \%$ for the $10 \mathrm{~mm}$ margin and $0.5 \%$ for the $15 \mathrm{~mm}$ margin. 


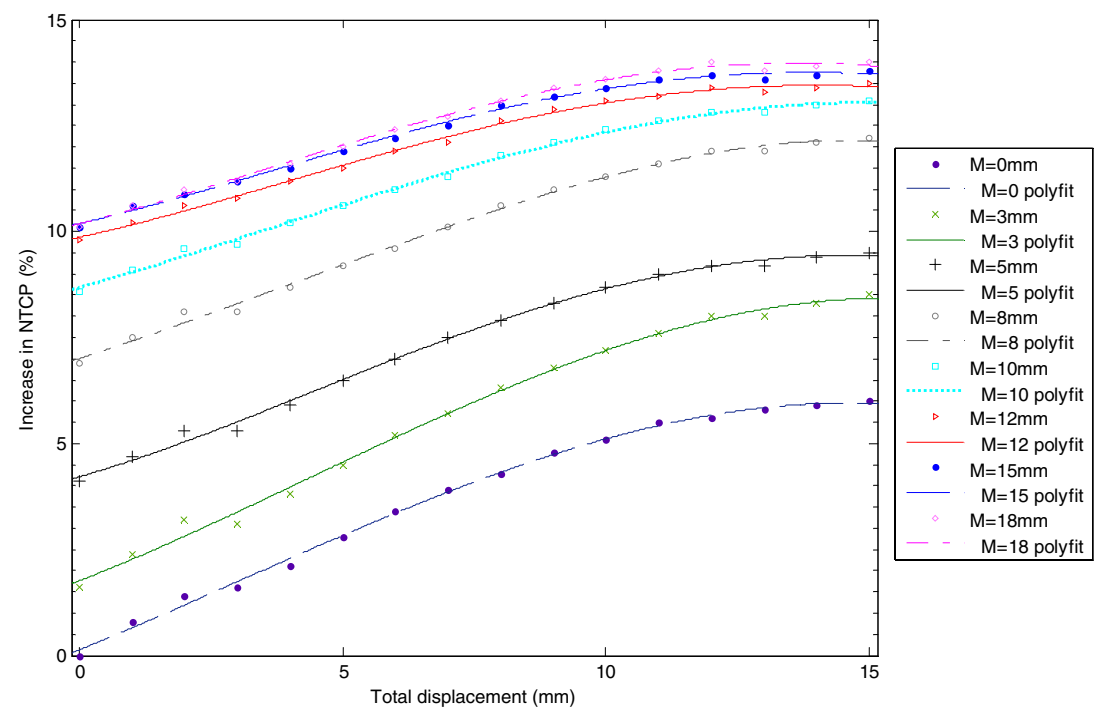

Figure 3. Effect of combined delineation, organ motion and set-up errors on rectal $\triangle \mathrm{NTCP}$, based on a 2 Gy per fraction dose prescription, for one patient's treatment plans using incremental margins $M$.

The increase in the treatment margin resulted in dose exposure to more of the rectal volume, and hence resulted in the step increase in NTCP, as shown in figure 3. The increase in the magnitude of the combined error further increased the NTCP values. The variation of $\triangle \mathrm{NTCP}$ with combined error was found to be approximately linear up to about $10 \mathrm{~mm}$ total displacement, and then nonlinear above that.

\subsection{Fuzzy logic system}

The Sugeno-type fuzzy system was chosen for modelling following initial experiments using both Sugeno and Mamdani-type fuzzy inference systems. From these initial experiments the Sugeno fuzzy inference system (FIS) gave results which predicted the expected output more closely. The other reason for using the Sugeno FIS was that outputs in the form of constants could be obtained. Figure 4 illustrates the basic operation of the Sugeno FIS using the input data ( $\triangle \mathrm{TCP}$ and $\triangle \mathrm{NTCP}$, as a function of radiotherapy errors), membership functions and fuzzy rules to compute the fuzzy output (PTV margin, as a function of $\triangle T C P$ and $\triangle$ NTCP).

The total number of membership functions and fuzzy rules used in this study were arrived at following an optimization procedure performed to improve the model accuracy. To do this the model was initiated using only a few membership functions and fuzzy rules and sequentially fine-tuned by increasing the number of functions and rules until the output function fulfilled the applied conditions.

The system consisted of two inputs, i.e. $\triangle \mathrm{TCP}$ and $\triangle \mathrm{NTCP}$, and one output, the PTV margin. The final model used in this study consisted of six membership functions which were defined for the inputs and output using linguistic terms, i.e. almost zero (AZ), very small (VS), small (S), medium (M), high (H) and very high (VH), to give the functions shown in figures 5(a) and (b). The Gaussian-type membership functions were chosen for modelling 


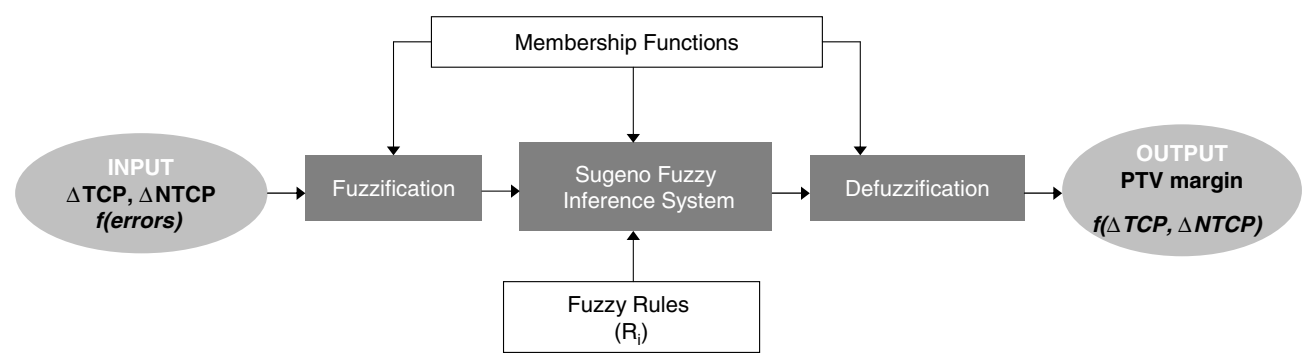

Figure 4. Basic operational principle of the Sugeno-type FIS used to calculate the CTV-PTV margin output function.

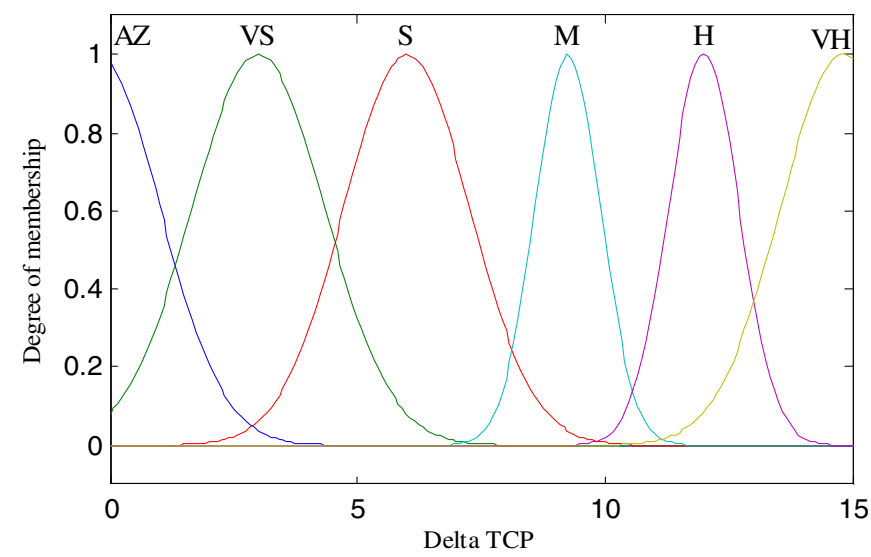

(a)

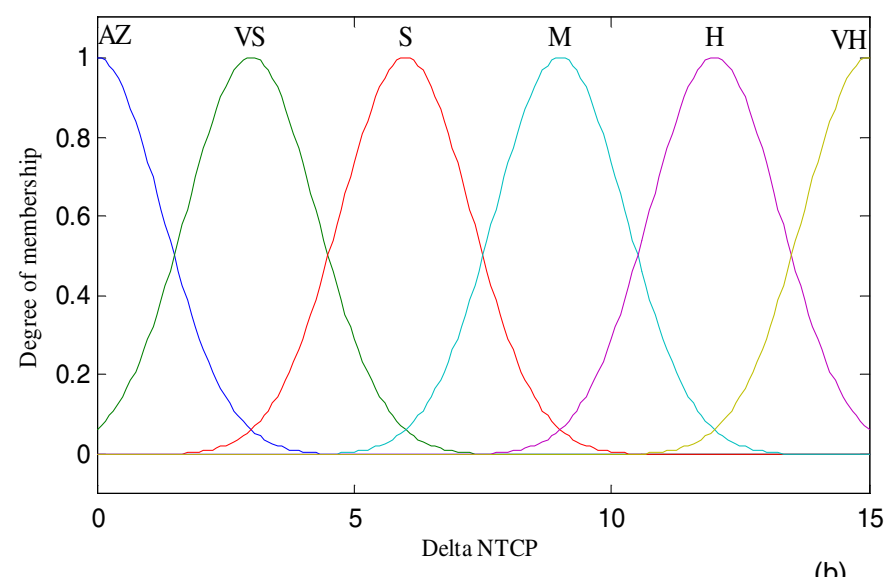

(b)

Figure 5. Membership functions for (a) $\triangle \mathrm{TCP}$, and (b) $\triangle \mathrm{NTCP}$ using six membership functions to describe the input terms; the widths of the functions were based on the gradient of the different sections of the input data and, for $\triangle \mathrm{NTCP}$, the critical organ tolerance doses. The functions were described as almost zero (AZ), very small (VS), small (S), medium (M), high $(\mathrm{H})$ and very high ( $\mathrm{VH})$. 
following an assessment of the output from different types of membership functions including triangular, trapezoidal and generalized bell. The 3D surface outputs from the non-Gaussian membership functions showed steep variations which imply uneven changes in CTV-PTV margin with TCP/NTCP changes, which did not correspond to the known relationships from the input data. The Gaussian plot showed relatively continuous and even transitions which agree well with the input data variation; hence, Gaussian membership functions were chosen for fuzzy modelling. Constant terms were used for the PTV margin membership functions.

Each rule in the Sugeno fuzzy system can be of the form

$$
\begin{aligned}
& R_{i}: \text { if }\left(x_{1} \text { is } f_{i 1}\right) \text { and } \ldots\left(x_{j} \text { is } f_{i j}\right) \ldots \text { and }\left(x_{m} \text { is } f_{i m}\right) \\
& \text { then } y_{i}=g_{i}\left(x_{1}, \ldots, x_{m}\right),
\end{aligned}
$$

where $i=1, n ; j=1, m, m$ is the number of inputs, $n$ is the number of rules; $x_{j}$ represents the $j$ th input; $f_{i j}$ is the membership function of the $i$ th rule; $y_{i}$ is the output of rule $R_{i}$ and $g_{i}$ represents the analytical function of the inputs $x_{j}$.

The fuzzy rules were formulated based on the assumptions that the loss in TCP due to organ displacement is compensated for by increasing the margin size whilst an increase in NTCP should be corrected for by reducing the PTV margin. An absolute NTCP value of $5 \%$ is considered to be the maximum acceptable tolerance for rectal complications (Emami et al 1991). The rules for the fuzzy system were also generated based on the conditions that for $\triangle \mathrm{NTCP}$ values above $5 \%$ the CTV-PTV margin was not allowed to exceed $10 \mathrm{~mm}$, and for $\triangle \mathrm{NTCP}$ above $10 \%$ the CTV-PTV margin was not permitted to exceed $5 \mathrm{~mm}$ to avoid rectal complications due to margin selection. Also, for a tubular structure such as the rectum, the irradiated fraction of the circumference is correlated to rectal bleeding (Bent et al 1993). Therefore the fraction of irradiated rectal wall was also calculated for each margin as a function of total displacement, and used in the formulation of the fuzzy membership rules. Permutations of the membership functions for $\triangle \mathrm{TCP}, \triangle \mathrm{NTCP}$ and PTV resulted in 36 fuzzy rules which are shown in the appendix.

During the defuzzification phase each input value was evaluated by a set of membership functions using each linguistic term in the function. The membership degree, i.e. nonzero response of each membership function and sequential operation of each fuzzy rule were used to generate the output of the Sugeno FIS. The final output $y(x)$ was calculated using the aggregated relative individual weighting adjusted factors using the centre-of-gravity defuzzification method given by

$$
y(x)=\frac{\sum_{i=1}^{n} \mu_{i} \omega_{i}}{\sum_{i=1}^{n} \omega_{i}},
$$

where $\mu_{i}$ is the weighting firing strength which determines the importance degree of each rule in the Sugeno FIS and $\omega_{i}$ is the output level of each rule. The centre of gravity defuzzification method was chosen for modelling because the crisp output obtained using this technique varies continuously when the input values also change continuously (van Broekhoven and de Baets 2006), as is the case with the input described in section 2.3.

\subsection{Convolution technique to optimize fuzzy output}

To provide a uniform and smooth transition in the output, a Gaussian convolution kernel (Fernández and Gutiérrez 2000, Anastassiou 2004) was applied to the Sugeno fuzzy output $y(x)$, to give the modified output $Y(x)$, according to the equation

$$
Y(x)=y(x) * G\left(x^{\prime}, y^{\prime} ; \sigma\right),
$$




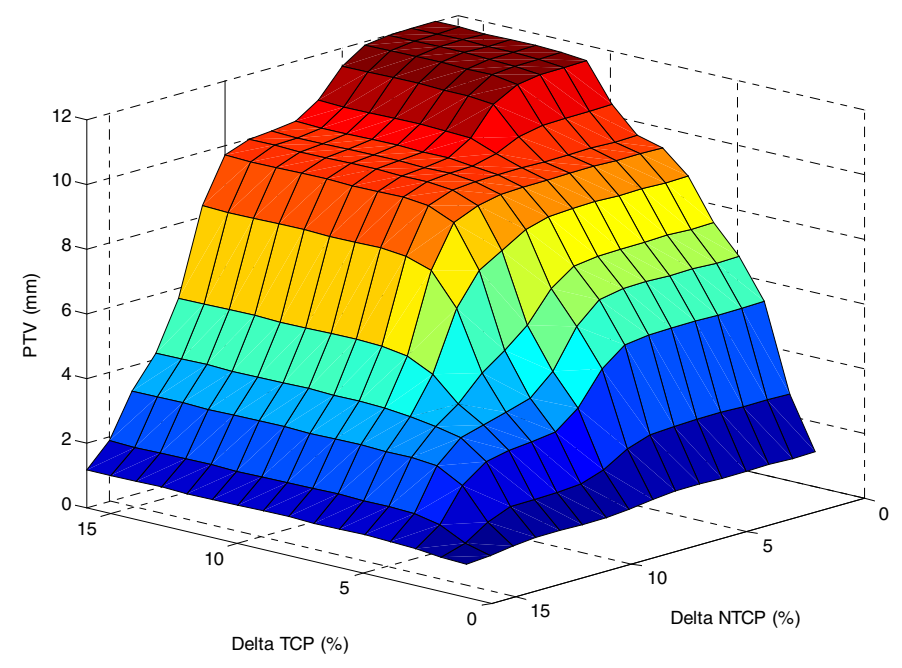

Figure 6. 3D output function from the fuzzy model. A course transition in the output function was observed.

where the Gaussian kernel $G\left(x^{\prime}, y^{\prime} ; \sigma\right)$ of width determined by the standard deviation $\sigma$ in two dimensions $x^{\prime}$ and $y^{\prime}$ is of the form

$$
G\left(x^{\prime}, y^{\prime}\right)=\frac{1}{2 \pi \sigma^{2}} \mathrm{e}^{-\frac{\left(x^{\prime}\right)^{2}+\left(y^{\prime}\right)^{2}}{2 \sigma^{2}}} .
$$

This kernel was chosen for its accuracy and parametric preservation properties as it retains the basic shape and point correspondences of the original data. It is also relatively simple to implement with fuzzy logic algorithms. Using this step the convolution transformation was applied to all elements of the initial Sugeno defuzzification output $y(x)$ with the expectation that the resulting output function would correspond better to the known variance in input parameters and reduce any unexpected steep gradients in the initial output surface.

\section{Results and discussion}

\subsection{Fuzzy output functions}

The output function was calculated initially for the Sugeno FIS and is shown in figure 6 as a $3 \mathrm{D}$ surface where each point corresponds to a specific $\triangle \mathrm{TCP}, \triangle \mathrm{NTCP}$ and PTV margin value. An increase in $\triangle$ NTCP results in a decrease in the PTV margin whilst an increase in $\triangle T C P$ corresponds to an increase in the PTV margin. In other words, as the loss in TCP increases the PTV margin is increased to compensate, and as damage to tissue increases the PTV margin is correspondingly reduced. A non-uniform variation in the output function was observed, with a pronounced step change in the region between $10 \mathrm{~mm}$ and $12 \mathrm{~mm}$ PTV margin. The output function however satisfied the applied rules and also the conditions regarding the 5\% and $10 \% \triangle \mathrm{NTCP}$ tolerances on the margin limitations. It was thus taken to be a good first approximation of the required output.

Application of the Gaussian convolution kernel to the Sugeno defuzzified output function resulted in a more uniform distribution and transition of the output function as shown in figure 7. This fine-tuned model is an improved representation which matches the observed 


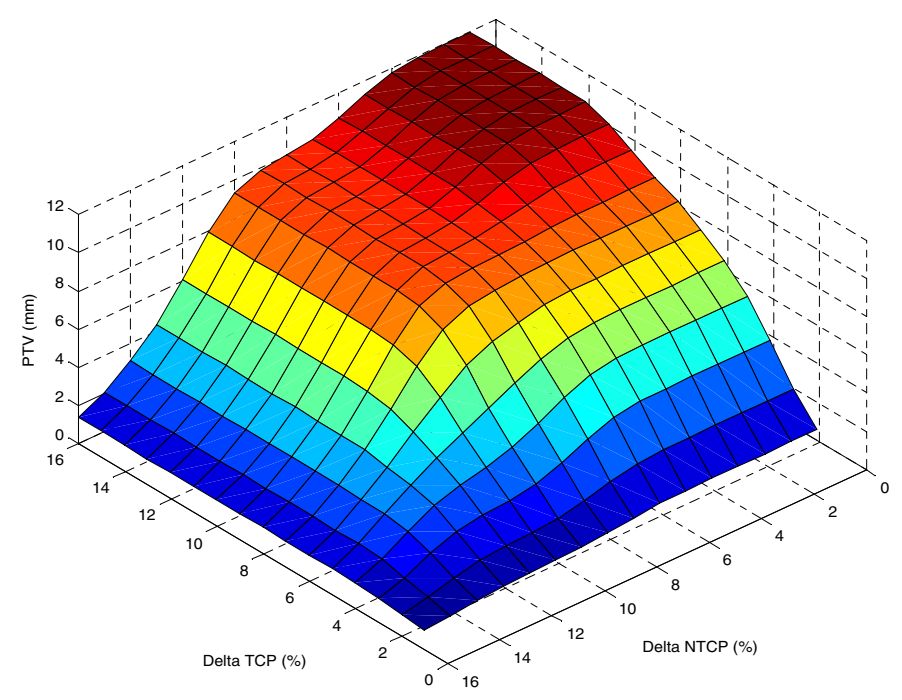

Figure 7. 3D output function following application of a Gaussian convolution kernel to initial fuzzy model output. The result shows a demonstrable improvement in the output function uniformity.

uniform transition in input and output parameters compared to the original Sugeno output function. Practically it was expected to result in the calculation of uniformly varying treatment margins. It also satisfied the applied rules and conditions previously stated.

In practice prior measurement or knowledge of the treatment uncertainties allows for the computation of $\triangle \mathrm{TCP}$ and $\triangle \mathrm{NTCP}$ using our dedicated software tool. The required CTV-PTV margin is then deduced from a matrix of the output function shown in figure 7 .

\subsection{Comparison to current margin formulation}

The CTV-PTV margin is currently derived based on margin recipe by van Herk et al (2000), and is calculated from the composite standard deviations of the systematic errors $(\Sigma)$ and the random errors $(\sigma)$ using the formulation $2.5 \Sigma+0.7 \sigma$. By using a technique of corresponding treatment errors as inputs, the CTV-PTV margin as derived by van Herk et al was compared to the corresponding fuzzy margin from our study. The CTV-PTV margin was calculated for a range of input systematic and random errors using van Herk et al's formula. Corresponding $\triangle \mathrm{TCP}$ and $\triangle \mathrm{NTCP}$ values due to the same systematic and random errors were computed and these derived parameters were then used to determine the corresponding CTV-PTV margin from the optimized fuzzy output. The van Herk-based and fuzzy-based PTV margins were then compared in terms of the root-mean-square of the standard deviation of incremental systematic and random errors, see figure 8. A standard uncertainty of $\pm 0.5 \mathrm{~mm}$ was computed for the error in the CTV-PTV margin values obtained using the fuzzy model in this study.

In relation to geometric effects, the fuzzy CTV-PTV margin from our study is best described by a third-order polynomial rather than a linear function. For total displacement standard errors ranging from 0 to $5 \mathrm{~mm}$ the fuzzy CTV-PTV margin was found to be on average $0.5 \mathrm{~mm}$ slightly larger than the van Herk-derived margin; however, taking the modelling uncertainty into account results in a good match between the margins. When the total displacement standard errors exceed $5.5 \mathrm{~mm}$, the fuzzy margin plateaus at $12 \mathrm{~mm}$. This is 


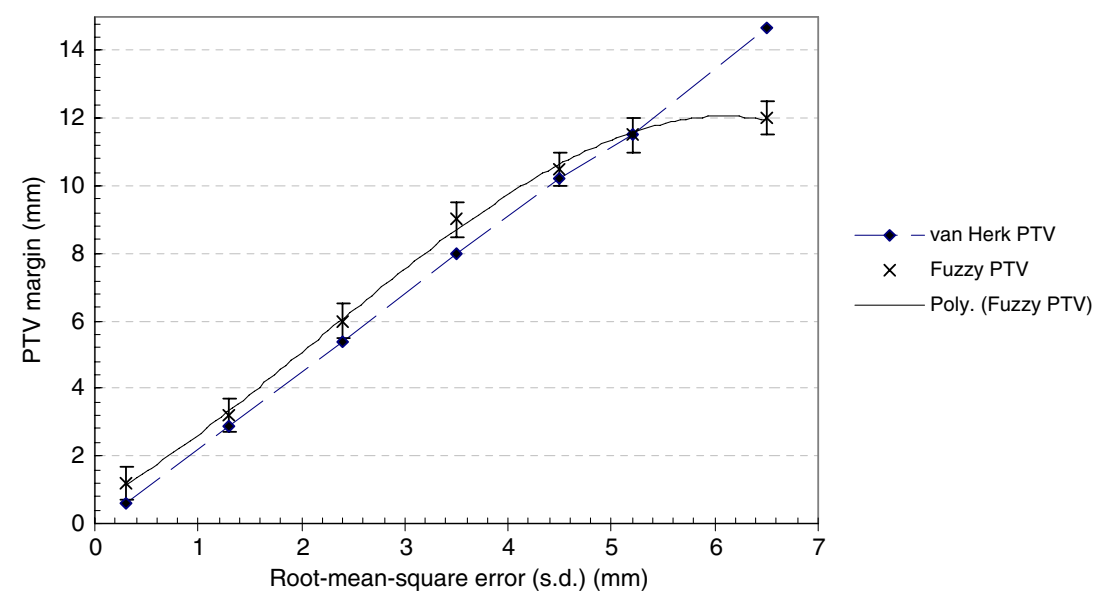

Figure 8. Comparison of van Herk-based and fuzzy model-based CTV-PTV margins, in terms of the standard deviation of total displacement errors, using the same corresponding uncertainties as inputs.

due to the effect of introducing TCP and NTCP in the margin formulation, and the dominance of the constraint for rectal sparing in the margin formulation. This variation is dependent on the chosen TCP and NTCP tolerances as well as the proximity between the tumour volume and the organs at risk. For the prostate organ a CTV-PTV margin of $10.0 \mathrm{~mm}$ is typically used for external beam radiotherapy treatment. The fuzzy PTV agrees very well with the van Herk-derived CTV-PTV at this margin region.

Whilst the van Herk formulation in theory shows a continuously linearly increasing CTVPTV margin, in practice the combined treatment errors encountered in prostate radiotherapy seldom result in CTV-PTV margins that exceed $12 \mathrm{~mm}$. In addition the CTV-PTV margin is reduced on the anterior rectal border to spare this organ. Using the fuzzy technique ensures that this information is embedded in the margin selection procedure, and the results explicitly present information that is otherwise assumed knowledge in radiotherapy treatment margin derivation.

\subsection{Application in IMRT treatment planning}

A currently active area of research in external beam radiotherapy is the reduction of treatment margins to spare organs at risk and healthy tissues whilst escalating the dose to improve tumour cure (Engelsman et al 2005, Zhang et al 2008). The effect of margin reduction was assessed for the fuzzy margin in comparison to currently used margins in IMRT treatment planning. A potential advantage of the fuzzy margin is the reduction in the possibility of missing cancerous cells in the target volume when margin reduction is applied as it is slightly bigger than current margins. The aim of this clinical investigation was to check if this characteristic would result in comparatively worse effects to the organs at risk.

The fuzzy-derived CTV-PTV margin was applied to a typical clinical example where the standard deviation from the systematic and random uncertainties is reduced to $3.0 \mathrm{~mm}$, i.e. assuming an appropriate IGRT correction protocol, such as cone beam CT (CBCT) imaging prior to treatment combined with a specific action level tolerance for set-up errors, has been used. In this case a van Herk-derived CTV-PTV margin of $6.5 \mathrm{~mm}$ would be used whilst 


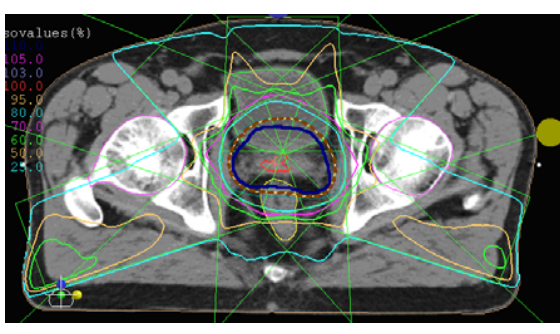

(a)

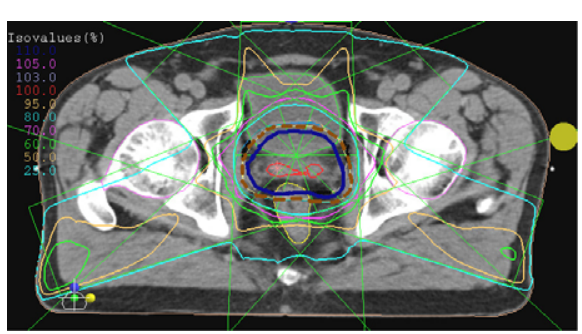

(b)

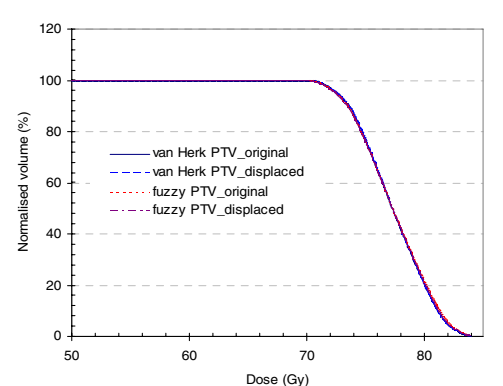

(c)

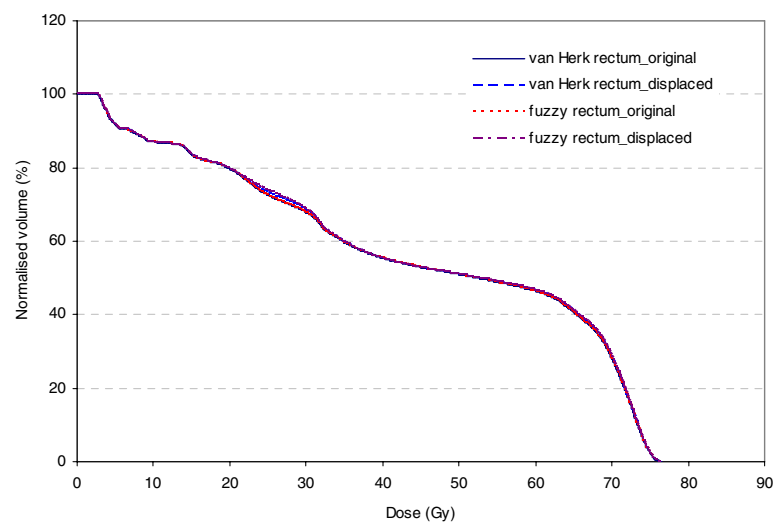

(d)

Figure 9. IMRT prostate treatment plans based on (a) van Herk CTV-PTV margin and (b) fuzzy CTV-PTV margin, using identical plan objectives and escalated dose prescription (dotted line shows the $95 \%$ isodose covering the PTV margin shown by the solid line), and the resultant mean dose volume histograms (DVHs) for the (c) prostate PTV and (d) rectum, showing the DVH variations after applying a displacement due to a systematic error of $3.0 \mathrm{~mm} \mathrm{s.d.} \mathrm{and} \mathrm{a} \mathrm{rotation} \mathrm{error}$ of $2^{\circ}$, assuming an IGRT correction protocol was used. No statistically significant differences were found between the DVHs of the target and critical organ doses between the original and displaced plans.

a fuzzy CTV-PTV margin of $7.0 \mathrm{~mm}$ would be used. Both margin sizes were used on the same IMRT prostate plan where the dose was escalated from 70 to $78 \mathrm{~Gy}$, see figure 9 . Equal displacement errors were applied to both treatment plans and the effect on the tumour and organs at risk, i.e. rectum, bladder and femoral heads, was assessed.

For the IMRT plans, as shown in figure 9, no significant differences were found in the prostate CTV, rectum, bladder and femoral heads' DVHs between the two plans when equal displacement errors were introduced. This is due to the small differences in these parameters in the original plans and the application of a reduced error magnitude due to the reduction of systematic and random errors from the assumed applied IGRT protocol. Table 2 summarizes the difference in the mean doses for the different structures of the two IMRT plans after applying the displacement. Comparing the mean dose difference parameters between the two plans again showed very similar values between the fuzzy margin based and the van Herkbased margin IMRT plan for the critical structures, i.e. the rectum and the bladder, whilst the target volume dose maintained good coverage. The slightly higher differences of up to $1.6 \%$ between the two plans were observed for the femoral heads; however, these were considered to be of very small dosimetrical significance. In addition the organ motion induced dose 
Table 2. Difference in mean dose between the original van Herk margin-based IMRT plan (VH1) and the displaced van Herk margin-based plan (VH2), as well as between the original fuzzy margin-based plan (FM1) and the displaced fuzzy margin-based plan (FM2), all based on 78 Gy dose prescription.

\begin{tabular}{|c|c|c|c|c|}
\hline \multirow[b]{2}{*}{ Organ } & \multicolumn{2}{|c|}{ Dose difference (cGy) } & \multicolumn{2}{|c|}{$\begin{array}{l}\text { Dose difference as a } \\
\text { percentage of mean } \\
\text { planned dose }(\%)\end{array}$} \\
\hline & $\begin{array}{l}\text { van Herk plans } \\
\text { (VH1-VH2) }\end{array}$ & $\begin{array}{l}\text { Fuzzy plans } \\
\text { (FM1-FM2) }\end{array}$ & $\begin{array}{l}\text { van Herk } \\
\text { plans }\end{array}$ & $\begin{array}{l}\text { Fuzzy } \\
\text { plans }\end{array}$ \\
\hline PTV & $3.13 \pm 0.1$ & $4.98 \pm 0.1$ & $0.0 \pm 0.01$ & $0.1 \pm 0.01$ \\
\hline Rectum & $18.67 \pm 0.5$ & $20.77 \pm 0.5$ & $0.4 \pm 0.02$ & $0.5 \pm 0.02$ \\
\hline Bladder & $13.34 \pm 0.5$ & $12.63 \pm 0.5$ & $0.3 \pm 0.02$ & $0.3 \pm 0.02$ \\
\hline Femoral head (left) & $220.38 \pm 5.0$ & $260.07 \pm 5.0$ & $8.0 \pm 0.2$ & $9.5 \pm 0.2$ \\
\hline Femoral head (right) & $172.65 \pm 5.0$ & $211.67 \pm 5.0$ & $7.2 \pm 0.2$ & $8.8 \pm 0.2$ \\
\hline
\end{tabular}

averaging effects in IMRT treatments would blur any dose differences between such plans resulting in near-identical delivered doses. Therefore use of the fuzzy model derived CTVPTV margin resulted in similar doses to currently used margins in IMRT treatment planning, even in the presence of treatment uncertainties.

The obtained results show that margins for radiotherapy use can be derived based not only on the geometrical considerations such as organ motion and set-up errors, as is common in most margin formulations, but also on the radiobiological effects of the radiotherapy errors, and fuzzy logic offers an intuitively straightforward modelling platform for the implementation of such a margin computation model. Other often missed effects such as delineation errors can be easily integrated into the model as shown.

\section{Conclusions}

Our results show the feasibility of applying a fuzzy convolution technique to derive margins for use in 3D conformal and IMRT radiotherapy planning. Good agreement was found between the margin derived using the fuzzy modelling technique and a commonly used margin formulation. Comparable plan parameters to current approaches were obtained when the margin was used in a dose escalated IMRT treatment planning example. The fuzzy margin proposed in this study can be derived for other treatment sites and individual patient cases due to anatomy variations. This should result in the derivation of patient-specific margins where required, whilst matching anatomies can utilize class solutions of the fuzzy margin derivation, which is proposed as a further extension of this work.

The radiobiological measures of response TCP and NTCP tend to be associated with high uncertainties; however, our results indicate that by using the changes in these parameters rather than their absolute values, margins which match well to current techniques can be derived and applied to give good results in IMRT treatment planning. The advantage of the proposed method is that it combines measured input/output data with radiobiological data and a priori knowledge using linguistic relationships in a computationally efficient model to produce results that explicitly express the assumed margin variation for treatment planning.

The limitations of treatment margins in accounting for all the uncertainties in radiotherapy also need to be understood. Changes in patient conditions such as weight loss and gain require the use of both image guidance techniques and deformable registration techniques to adjust the 
treatment margins. Reflex conditions such as the patient coughing or swallowing which were previously difficult to correct for can now be solved using gating and tracking techniques. The fuzzy model used in this study can potentially be easily combined with deformable registration, gating and tracking algorithms to provide an optimized margin tool for treatment planning.

\section{Acknowledgments}

We would like to express our appreciation to Tony Palmer, Head of Radiotherapy Physics at Portsmouth, for helpful comments and discussions. This work was financially supported by the Medical Physics Department of Portsmouth Hospitals NHS Trust (UK).

\section{Appendix}

The fuzzy system used in our study, based on six membership functions each for $\triangle \mathrm{TCP}$, $\triangle$ NTCP and PTV margin as well as applied radiobiological tolerance conditions, resulted in the formulation of a total of 36 rules for use in the fuzzy model. These rules are shown below.

(i) If $\triangle \mathrm{TCP}$ is almost zero and $\triangle \mathrm{NTCP}$ is almost zero then PTV is almost zero.

(ii) If $\triangle \mathrm{TCP}$ is very small and $\triangle \mathrm{NTCP}$ is almost zero then PTV is small.

(iii) If $\triangle \mathrm{TCP}$ is very small and $\triangle \mathrm{NTCP}$ is very small then PTV is small.

(iv) If $\triangle \mathrm{TCP}$ is small and $\triangle \mathrm{NTCP}$ is small then PTV is medium.

(v) If $\triangle \mathrm{TCP}$ is medium and $\triangle \mathrm{NTCP}$ is very small then PTV is high.

(vi) If $\triangle \mathrm{TCP}$ is small and $\triangle \mathrm{NTCP}$ is medium then PTV is medium.

(vii) If $\triangle T C P$ is medium and $\triangle N T C P$ is medium then PTV is medium.

(viii) If $\triangle \mathrm{TCP}$ is medium and $\triangle \mathrm{NTCP}$ is high then PTV is small.

(ix) If $\triangle \mathrm{TCP}$ is high and $\triangle \mathrm{NTCP}$ is high then PTV is very small.

(x) If $\triangle \mathrm{TCP}$ is high and $\triangle \mathrm{NTCP}$ is very high then PTV is almost zero.

(xi) If $\triangle \mathrm{TCP}$ is very high and $\triangle \mathrm{NTCP}$ is very high then PTV is almost zero.

(xii) If $\triangle \mathrm{TCP}$ is very small and $\triangle \mathrm{NTCP}$ is small then PTV is small.

(xiii) If $\triangle \mathrm{TCP}$ is small and $\triangle \mathrm{NTCP}$ is very small then PTV is medium.

(xiv) If $\triangle T C P$ is very high and $\triangle N T C P$ is high then PTV is high.

(xv) If $\triangle \mathrm{TCP}$ is medium and $\triangle \mathrm{NTCP}$ is small then PTV is medium.

(xvi) If $\triangle \mathrm{TCP}$ is very high and $\triangle \mathrm{NTCP}$ is high then PTV is very small.

(xvii) If $\triangle \mathrm{TCP}$ is very high and $\triangle \mathrm{NTCP}$ is medium then PTV is medium.

(xviii) If $\triangle \mathrm{TCP}$ is almost zero and $\triangle \mathrm{NTCP}$ is small then PTV is almost zero.

(xix) If $\triangle \mathrm{TCP}$ is almost zero and $\triangle \mathrm{NTCP}$ is medium then PTV is almost zero.

(xx) If $\triangle \mathrm{TCP}$ is small and $\triangle \mathrm{NTCP}$ is medium then PTV is small.

(xxi) If $\triangle \mathrm{TCP}$ is small and $\triangle \mathrm{NTCP}$ is almost zero then PTV is medium.

(xxii) If $\triangle \mathrm{TCP}$ is medium and $\triangle \mathrm{NTCP}$ is very small then PTV is high.

(xxiii) If $\triangle \mathrm{TCP}$ is high and $\triangle \mathrm{NTCP}$ is small then PTV is medium.

(xxiv) If $\triangle \mathrm{TCP}$ is very high and $\triangle \mathrm{NTCP}$ is small then PTV is medium.

(xxv) If $\triangle \mathrm{TCP}$ is almost zero and $\triangle \mathrm{NTCP}$ is very small then PTV is almost zero.

(xxvi) If $\triangle \mathrm{TCP}$ is almost zero and $\triangle \mathrm{NTCP}$ is high then PTV is almost zero.

(xxvii) If $\triangle \mathrm{TCP}$ is very small and $\triangle \mathrm{NTCP}$ is high then PTV is very small.

(xxviii) If $\triangle \mathrm{TCP}$ is small and $\triangle \mathrm{NTCP}$ is high then PTV is very small.

(xxix) If $\triangle \mathrm{TCP}$ is high and $\triangle \mathrm{NTCP}$ is almost zero then PTV is high.

(xxx) If $\triangle \mathrm{TCP}$ is very high and $\triangle \mathrm{NTCP}$ is very small then PTV is high.

(xxxi) If $\triangle \mathrm{TCP}$ is almost zero and $\triangle \mathrm{NTCP}$ is very high then PTV is almost zero.

(xxxii) If $\triangle \mathrm{TCP}$ is very small and $\triangle \mathrm{NTCP}$ is very high then PTV is almost zero. 
(xxxiii) If $\triangle \mathrm{TCP}$ is high and $\triangle \mathrm{NTCP}$ is almost zero then PTV is high.

(xxxiv) If $\triangle \mathrm{TCP}$ is very high and $\triangle \mathrm{NTCP}$ is high then PTV is high.

(xxxv) If $\triangle \mathrm{TCP}$ is small and $\triangle \mathrm{NTCP}$ is very high then PTV is almost zero.

(xxxvi) If $\triangle \mathrm{TCP}$ is medium and $\triangle \mathrm{NTCP}$ is very high then PTV is almost zero.

\section{References}

Anastassiou G A 2004 Fuzzy approximation by fuzzy convolution type operators Comput. Math. Appl. 48 1369-86 Bent V A, Adams J A, Shipley W U, Urie M M, McNamus P L, Efird J T, Willett C G and Goiten M 1993 Late rectal bleeding following combined X-ray and photon high dose irradiation for patients with stages T3-T4 prostate carcinoma Int. J. Radiat. Oncol. Biol. Phys. 26 551-7

Burman C, Kutcher G J, Emami B and Goitein M 1991 Fitting of normal tissue tolerance data to an analytic function Int. J. Radiat. Oncol. Biol. Phys. 21 123-35

Emami B et al 1991 Tolerance of normal tissue to therapeutic irradiation Int. J. Radiat. Oncol. Biol. Phys. 21 109-22

Engelsman M, Sharp G C, Bortfeld T, Onimaru R and Shirato H 2005 How much margin reduction is possible through gating or breath hold? Phys. Med. Biol. 50 277-490

Fernández F and Gutiérrez J 2000 Specification and optimization of fuzzy systems using convolution techniques Signal Process. 80 935-49

Gay H A and Niermierko A 2007 A free program for calculating EUD-based NTCP and TCP in external beam radiotherapy Phys. Medica 23 115-25

ICRU 1993 Prescribing, recording and reporting photon beam therapy ICRU Report 50 (Bethesda, MD: International Commission on Radiation Units and Measurements)

ICRU 1999 Prescribing, recording and reporting photon beam therapy (supplement to ICRU Report 50) ICRU Report 62 (Bethesda, MD: International Commission on Radiation Units and Measurements)

Jones D, Hafermann M D, Rieke J W and Vermeulen S S 1995 An estimate of the margin required when defining blocks around the prostate Br. J. Radiol. 68 740-6

Kim E, Lee H, Park M and Park M 1998 A simply identified Sugeno-type fuzzy model via double clustering Inf. Sci. 110 25-39

Kobashi S, Fujimoto Y, Ogawa M, Ando K, Ishikura R, Imawaki S, Hirota S and Hata Y 2009 Fuzzy logic assisted quantification of gyral deformation index using magnetic resonance images for the infantile brain Proc. IEEE 39th Int. Symp. on Multiple-Valued Logic pp 24-9

Lind B, Kallman P, Sundelin B and Brahme A 1993 Optimal radiation beam profiles considering uncertainties in beam patient alignment Acta Oncol. 32 331-42

Löf J, Lind B K and Brahme A 1995 Optimal radiation beam profiles considering the stochastic process of patient positioning in fractionated radiation therapy Inverse Problems 11 1189-209

Miralbell R, Nouet P, Rouzaud M, Bardina A, Hejira N and Schneider D 1998 Radiotherapy of bladder cancer: relevance of bladder volume changes in planning boost treatment Int. J. Radiat. Oncol. Biol. Phys. 41 741-6

Ragazzi G, Mangili P, Fiorino C, Cattaneo G M, Bolognesi A, Reni M and Calandrino R 1997 Variations of tumour control and rectum complication probabilities due to random set-up variations during conformal radiation therapy of prostate cancer Radiother. Oncol. 44 259-63

Seddon B, Bidmead M, Wilson J, Khoo V and Dearnaley D 2000 Target volume definition in conformal radiotherapy for prostate cancer: quality assurance in the MRC RT-01 trial Radiother. Oncol. 56 73-83

Stavrev P V, Stavreva N A and Round W H 1996 A new method for optimum dose distribution determination taking tumour mobility into account Phys. Med. Biol. 41 1679-89

Stroom J C, de Boer H C J, Huizenga H and Vissier A G 1999 Inclusion of geometrical uncertainties in radiotherapy treatment by means of coverage probability Int. J. Radiat. Oncol. Biol. Phys. 43 905-19

Sur R K, Clinkard J, Jones W G, Taylor R E, Close H J, Chaturvedi A and Carey B 1993 Changes in target volume during radiotherapy treatment of invasive bladder carcinoma Clin. Oncol. 5 30-3

van Broekhoven E and de Baets B 2006 Fast and accurate center of gravity defuzzification of fuzzy system outputs defined on trapezoidal fuzzy partitions Fuzzy Sets Syst. 157 904-18

van Herk M, Remeijer P, Rasch C and Lebesque J V 2000 The probability of correct target dose: dose-population histograms for deriving treatment margins in radiotherapy Int. J. Radiat. Oncol. Biol. Phys. 47 1121-35

Waschek T, Levegrun S, van Kampen M, Glesner M, Engenhart-Cabillic R and Schlegel W 1997 Determination of target volumes for three-dimensional radiotherapy of cancer patients with a fuzzy system Fuzzy Sets Syst. 89 361-70

Wolbarst A B, Sternick E S, Curran B H, Kosinski R J and Dritschilo A 1980 Optimized radiotherapy treatment planning using the complication probability factor (CPF) Int. J. Radiat. Oncol. Biol. Phys. 6 723-8 
Wu Q, Mohan R, Niermerko A and Schmidt-Ullrich R 2002 Optimization of intensity-modulated radiotherapy plans based on the equivalent uniform dose Int. J. Radiat. Oncol. Biol. Phys. 52 224-35

Ying H 1998 The Takani-Sugeno fuzzy controllers using the simplified linear control rules are non-linear variable gain controllers Automatica 34 157-67

Zhang Z, Hu W, Zhu J, Li G, Ma X and Ye J 2008 Margin reduction with active breathing control in image guided gastric cancer radiotherapy Int. J. Radiat. Oncol. Biol. Phys. 72 S255-6 